\title{
Collaborative Localization: Enhancing WiFi-Based Position Estimation with Neighborhood Links in Clusters
}

\author{
Li-wei Chan, Ji-rung Chiang, Yi-chao Chen, Chia-nan Ke, Jane Hsu, \\ and Hao-hua Chu \\ Graduate Institute of Networking and Multimedia, \\ Department of Computer Science and Information Engineering, \\ National Taiwan University \\ \{yjhsu, hchu\}@csie.ntu.edu.tw
}

\begin{abstract}
Location-aware services can benefit from accurate and reliable indoor location tracking. The widespread adoption of $802.11 \mathrm{x}$ wireless LAN as the network infrastructure creates the opportunity to deploy WiFi-based location services with few additional hardware costs. While recent research has demonstrated adequate performance, localization error increases significantly in crowded and dynamic situations due to electromagnetic interferences. This paper proposes collaborative localization as an approach to enhance position estimation by leveraging more accurate location information from nearby neighbors within the same cluster. The current implementation utilizes ZigBee radio as the neighbor-detection sensor. This paper introduces the basic model and algorithm for collaborative localization. We also report experiments to evaluate its performance under a variety of clustering scenarios. Our results have shown 28.2-56\% accuracy improvement over the baseline system Ekahau, a commercial WiFi localization system.
\end{abstract}

\section{Introduction}

Technologies for indoor location tracking are important for deploying location-aware services in public buildings like museums, transit stations, or hospitals. For example, visitors can receive background information about the exhibit they are viewing, passengers can obtain real-time status update on their next connections, and emergency medical personnel can locate critical patients or equipments. Given accurate and reliable location information, an intelligent museum guide can provide museum visitors with relevant information and timely services. In contrast, location errors may result in undesirable deliveries of the wrong information to the wrong people at the wrong place.

The widespread adoption of $802.11 \mathrm{x}$ wireless LAN as a common network infrastructure enables WiFi-based localization with few additional hardware costs. Microsoft Research proposed an RF-based indoor location tracking system by processing signal strength information at multiple base stations [1]. Since then, much research has focused on improving WiFi-based localization from noisy signals, and has achieved position estimation with up to $90 \%$ accuracy within an error of 1 meter. While such performance is sufficient for most indoor pervasive computing 
applications, the results are somewhat misleading since they are usually measured in static, ideal situations with minimal signal interference. Our previous work [12] demonstrated the impact on position estimation with people moving around a target mobile device. Not only does the estimated position become unstable, but the error also rises twofold due to electromagnetic interferences by the human body.

People cluster naturally in typical social settings. Based on observing museum visits, people often browse through the exhibits with their family or friends, forming relatively stable moving clusters. Visitors also tend to gather in front of popular exhibits, instantly creating temporary static clusters. Figure 1, two random snapshots taken at the National Museum of Natural Science, illustrates that most visitors are in the midst of small crowds. In addition, clusters are dynamic. First, they may move as a group with varying speeds. Second, they may assemble and disassemble over time. Similar scenarios happen frequently in other public places, such as passengers departing or arriving in transit stations.
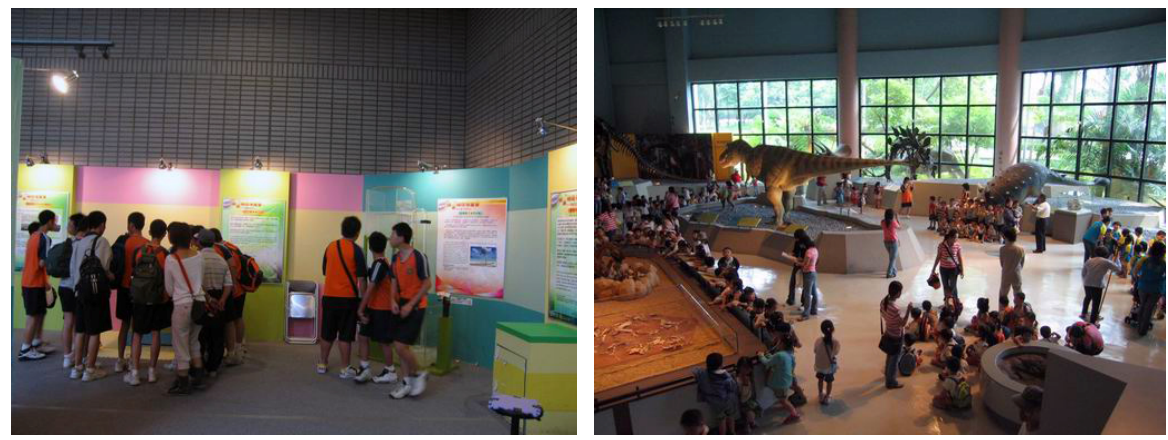

Fig. 1. Visitors at the National Museum of Natural Science in Taiwan

This research investigates the problem of WiFi-based localization in clustering scenarios. This paper starts by analyzing the effects on positioning errors due to human clusters of varying sizes. We then propose collaborative localization as an approach to improving position estimation accuracy by leveraging potentially more accurate position information from nearby neighbors. Section 3 introduces the basic idea and models supporting collaborative localization. Section 4 presents the experimental results showing the advantages of the proposed approach. Section 5 provides pointers to related research, followed by the conclusion and future work in Section 6.

\section{Clustering}

A traditional WiFi-based location system utilizes wireless signal strength to estimate locations in two phases. First, the offline training phase collects Received Signal Strength Indicator (RSSI) from multiple access points (APs) at each sampled location. The results are saved in a radio map. Second, the online estimation phase matches the RSSI from a target mobile device to each sampled location on the radio map. The coordinates of the target location can be estimated deterministically or probabilistically. To 
understand the impact of human clusters on location accuracy, we have conducted preliminary experiments described in our previous work [12]. Results of these experiments have shown that human clustering can create strong interferences with surrounding signals, leading to significant degradation in location accuracy. In a case of six people walking around a user carrying the target mobile device, the position estimated by the Ekahau location system [4] becomes unstable, leading to doubling of the average positioning error.

Several recent localization systems [11][12] have worked on the challenge of providing stable position estimation under different environmental dynamics, including change of floor layout, change in relative humidity, and moving people. Since major floor layout changes occur less frequently, they can be managed by rebuilding a selective part of a radio map. To adapt to different relative humidity levels, a separate radio map can also be constructed for each humidity level. However, coping with moving people is more challenging, because it is infeasible to model and enumerate all possible cases of human clustering formations, human orientations and moving speeds, and further, to construct corresponding radio maps. To our knowledge, we have not found any satisfactory solution that can address the challenge of people dynamics.

To measure quantitatively the impact of people clustering on the amount of degradation in positioning accuracy, we have conducted experiments, again using Ekahau as an example. For each test, users stand at pre-specified positions to form clusters of sizes 1, 3, and 7 person(s). Each user carries a Notebook PC equipped with a wireless network card to collect RSSIs from APs. The same WiFi cards are used to minimize errors due to different signal strength interpretations by different WiFi card drivers. The results are plotted in Figure 2, showing that the positioning accuracy degrades significantly with an increasing cluster size. In a single person case (no clustering), Ekahau can achieve a high positioning accuracy of approximately $80 \%$ within an error of 2 meters. In comparison, Ekahau's positioning accuracy degrades to $60 \%$ in the case of 3-person clusters, and further degrades to less than $30 \%$ in the case of 7 person clusters. The general trend is that increasing cluster size leads to rapidly decreasing average positioning accuracy and precision.

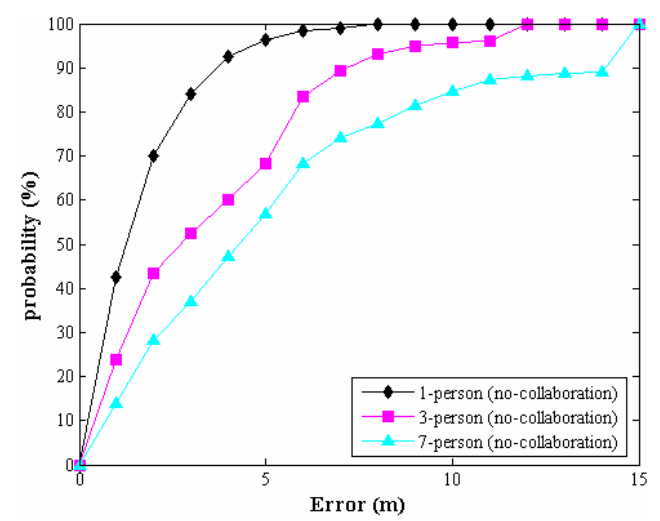

Fig. 2. CDF of the cluster's average positioning errors 
To investigate how clustering influences the positioning estimation accuracy for each individual in a cluster, we have plotted cumulative density functions (CDFs) of average positioning errors experienced by each individual in Figure 3. It shows a 7person clustering case where each colored curve represents the positioning accuracy experienced by one person in a cluster. The relative position of each person in a cluster is shown in a small diagram at the bottom. Although clustering degrades average positioning accuracy of a cluster (shown in Figure 2), the amount of degradation experienced by people varies within the same cluster. In the 7-person clustering case shown in Figure 3, user-7's accuracy is almost unaffected, whereas user-3's accuracy is significantly reduced.

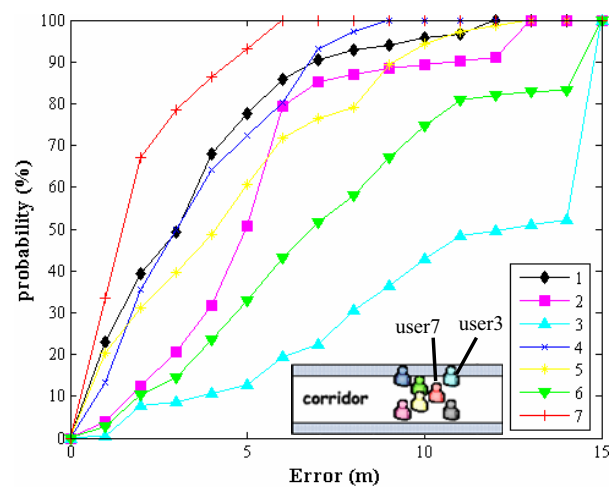

Fig. 3. CDFs of each node's average positioning error within a 7-person cluster

The next question is what causes such large variance in positioning accuracy among individuals within the same cluster? We have found several possible direct and indirect causes, such as people's relative position within a cluster, their orientation, the way (e.g., the height) they hold the device, the geometry of the environment, etc. Rather than considering clustering as a hindrance to improving accuracy in localization systems, we turn them into an advantage by exploiting collaboration among neighbor nodes.

\section{Design and Implementation}

We propose collaborative localization to leverage the variance in location accuracy among nodes within a cluster. Intuitively, nodes in the same cluster may help localize each other so as to enhance the overall average positioning accuracy of the cluster. By identifying nodes with high location accuracy, we can use their location estimations to help better localize neighbor nodes with lower location accuracy. The design for collaborative localization is shown in Figure 4. It consists of the following three modules: Neighborhood Detection, Confidence Estimation, and Collaborative Error Correction. The general work flow of the system is summarized as follows. 
1. Neighborhood Detection identifies nearby neighbor nodes as possible candidates for collaborative localization;

2. Confidence Estimation computes and attaches a confidence score to the position estimation returned by a given localization system (e.g., Ekahau). Confidence measures the probability of a location estimation being accurate, and it will be formally defined in Section 3.2.

3. Collaborative Error Correction adjusts the estimated location of the target node using the estimated locations of neighboring nodes with higher confidence scores. This way, the error in location estimation of the target node can be reduced.

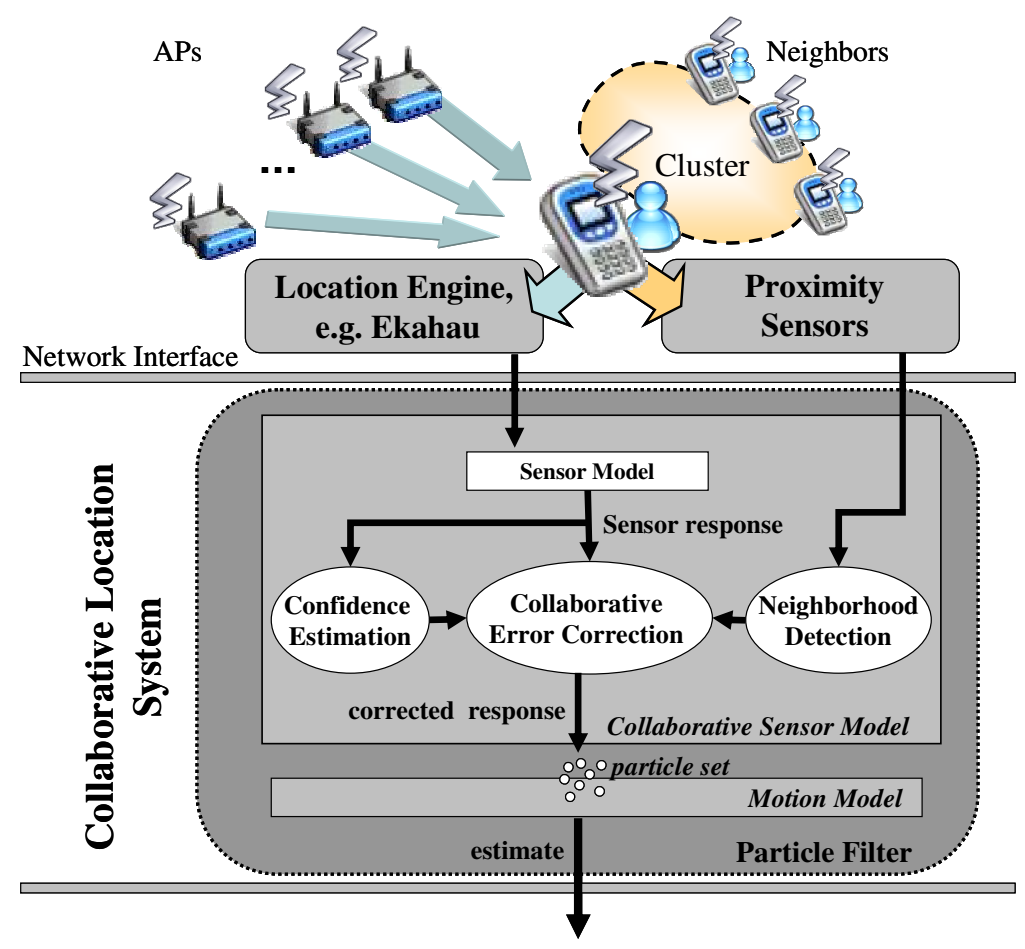

Fig. 4. Design of collaborative location system

\subsection{Neighborhood Detection}

For each target node, the Neighborhood Detection finds its neighbor nodes within a certain proximity radius (e.g., 2 meters). Each node periodically probes its neighborhood through a proximity sensor, and the system continues to track the neighboring relationships among all target nodes.

We experimented with a number of proximity sensors, including Bluetooth, ZigBee, infrared, and ultra-sound, and have chosen ZigBee in our implementation. ZigBee offers the following advantages: (1) ZigBee's signal strength decays quickly over a relatively short distance, so its signal strength signatures can be mapped accurately 
to proximity distances within a range of $1 \sim 2$ meters; (2) The simplicity of ZigBee protocol supports instantaneous connection, which facilitates proximity detection based on signal strength; (3) ZigBee does not suffer from the line-of-sight problem as with infrared sensors, so users can carry their WiFi nodes in their pockets; and (4) ZigBee radio has relatively low power consumption.

In our current implementation, the neighborhood detection is defined in terms of the actual distance calculated from the ground truths of the target nodes. A neighbor node is defined as having a proximity distance of less than 2 meters to the target node.

\subsection{Confidence Estimation}

Confidence Estimation measures the probability of the location estimation, obtained from an underlying localization engine, being close to its true location. In other words, a high (low) confidence score implies that the location estimation has a high (low) probability of being the true location. Confidence in location estimation correlates highly to positioning stability of a target node computed over time from a particle filter. Location estimation is based on the sensor model generated by the Ekahau localization engine, which is used in conjunction with a motion model to constrain location estimation within a reasonable variation consistent with human movement. That is, given the current location of a target, there is a limited range of possible locations that a human may reach. As a result, the difference between the location estimated from a sensor response $S$ and the bounded estimation $P$ returned from a particle filter implies the uncertainty in location estimation. If the position of a target node changes beyond what's prescribed by the motion model, a low confidence score is assigned.

The confidence estimation can be derived by accumulating successive uncertainties over a specified time window. Specifically, we define the confidence at time $t$ according to the following equation:

$$
\operatorname{Conf}(t)=e^{\frac{-\left[\sum_{i=0}^{S} w(i) \cdot u c(t-i)\right]^{2}}{k}} .
$$

Here, $t$ is the current time stamp, $i$ is an accumulation index, and $s$ is the length of the time window. Let $w(i)$ be the weight to accumulate uncertainties at different times within the window, and $u c(t-i)$ measure the uncertainty of a sensor response, i.e. the difference between the location estimation from the sensor response and the bounded estimation returned from a particle filter at time $(t-i)$. Equation (1) computes the weighted sum of uncertainties over an accumulation window $s$, normalizing it to a value between $[0,1]$. The value $k$ is a constant that adjusts the speed of decline in a logarithmic curve - a higher $k$ value means that the curve will decline more slowly. A high confidence score, e.g., 0.95, means that a particle filter has found little uncertainty over the time window, indicating high accuracy in location estimation. In the current implementation, $s$ is defined as the 3 most recent samples, constant $k$ is 300 , and the weight $w(i)$ is equal for the three samples. 


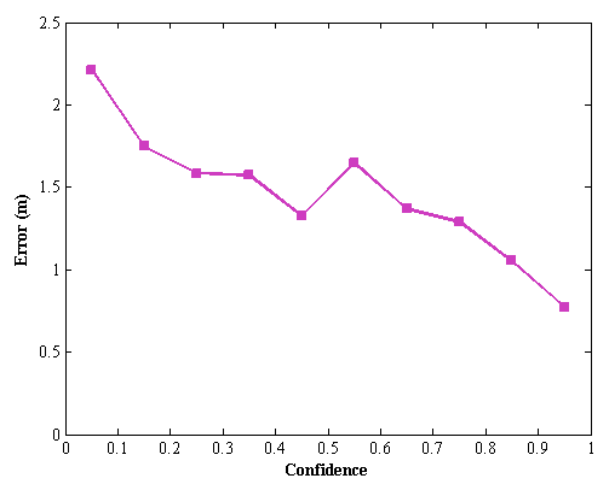

Fig. 5. Confidence scores and location estimation errors

In order to validate how well Equation (1) models the relationship between confidence and accuracy of position estimations, we have conducted an experiment by collecting 1179 location estimation samples. These samples' confidence scores are computed from Equation (1) and then plotted against their estimation errors from their true locations. Results in Figure 5 show a good inverse relationship between confidence and error.

Other applications can be created using the framework illustrated in Figure 4. Since a confidence estimation is independent of sensor models, a different localization algorithm (e.g., a simple nearest neighbor algorithm) can be used in a sensor model. At the same time, a different confidence function that is more accurate than the one described in Equator (1) can be used.

\subsection{Collaborative Error Correction}

Collaboration Error Correction (CEC) enhances location estimation from particles of a target node by removing estimation that has a lower confidence score, from estimations of its neighbor nodes that have higher confidence scores. In order to produce this enhancement, the collaborative error correction step requires the following information: (1) a set of neighbor nodes within proximity of a target node $N_{s}=\left\{N_{l}, N_{2}, . . N_{s}\right\}$ detected from the neighborhood detection, and (2) a set of pairs of their location estimations and confidence scores $\left.\left\{\left\langle N_{1}{ }^{\text {pos }}, N_{1}{ }^{\text {conf }}\right\rangle,\left\langle N_{2}{ }^{\text {pos }}, N_{2}{ }^{\text {conf }}\right\rangle, . .<N_{s}^{\text {pos }}, N_{s}{ }^{\text {conf }}\right\rangle\right\}$.

Collaborative enhancement is based on the concept of attraction from magnetic interactions in nature. A high confidence node $N_{x}$, whose location estimation is at $N_{x}{ }^{p o s}$, is assigned a stronger magnetic charge $N_{x}{ }^{\text {conf }}$. On the other hand, a low confidence neighbor node $N_{y}$, whose location estimation is at $N_{y}{ }^{p o s}$, is assigned a weaker magnetic charge $N_{y}{ }^{\text {conf }}$. Based on natural magnetic interactions, a low confidence node, acting as a nail, will be pulled from its original position at $N_{y}{ }^{p o s}$ toward the position of a high confidence node at $N_{x}{ }^{p o s}$. The magnitude of this attraction force (refer to as the neighboring force) is proportional to the ratio $N_{x}{ }^{\text {conf }} / N_{y}{ }^{\text {conf }}$.

The actual mechanism can be described as follows. In step 1 , for each node $N$, we collect its proximity nodes and <estimated location, confidence score $>$ pairs. In 
step 2, the neighboring force $F_{b}$ between a target node $N$ and one of its neighbor node $N_{b}$, is computed as follows:

$$
F_{b}=\frac{N_{b}^{\text {Conf }}}{N^{\text {Conf }}+N_{b}^{\text {Conf }}} \times\left|D\left(N^{\text {pos }}, N_{b}{ }^{\text {pos }}\right)-r \times(1+\varepsilon)\right| \times u\left(N^{\text {pos }}-N_{b}{ }^{\text {pos }}\right)
$$

Here, $r$ measures the proximity distance between the node pairs, $\varepsilon$ is a constant measuring the amount of error ratio in a neighbor proximity measurement, $D$ is the Euclidean distance between two coordinates $N^{\text {pos }}$ (a target node's position) and $N_{b}^{\text {pos }}$ (a neighbor node's position), and the unit vector $u\left(N_{b}{ }^{\text {pos }}-N^{\text {pos }}\right)$ gives the direction of this neighboring force. In step 3, since a target node can have multiple neighbor nodes, individual attraction forces contributed from each of its neighbor nodes are summed into an aggregate neighboring force $F$, which is defined in equation (3). Note that $F$ is computed as a weighted sum of neighboring forces, with the weight equal to the normalized confidence level of each of its contributing neighbor nodes.

$$
F=\sum_{b=1}^{s} \frac{N_{b}^{\text {Conf }}}{\sum_{i=1}^{s} N_{i}^{\text {Conf }}} \times F_{b} .
$$

In the last step, we apply $F$ to correct the location estimation of a target node. This corrected location estimation is then used to assign probabilities of particles. Finally, the particle with the highest probability is chosen as location estimation.

\section{Experimental Results}

The following experiments were performed on the corridors of the $3^{\text {rd }}$ floor of the Computer Science Department building in our university as shown in Figure 6. The baseline WiFi positioning engine is a commercial product Ekahau [4]. All users brought mobile devices equipped with the same brand IEEE 802.11g WLAN card.

\subsection{Neighborhood Sensing}

In the real environments, errors caused by neighborhood sensing technology will reduce benefit generated by the proposed algorithm. The error model of the neighborhood detection is derived from ZigBee radio, which is the chosen sensor. In neighborhood detection, each radio periodically transmits and receives signals from neighbor radios. From the received signals, their RSSI values are measured. Figure 7(a) shows that RSSI decays rapidly when distance between two radios increases from 1 to 3 meters, which is an ideal range for neighbor proximity detection. We adapt a simple method to detect neighbors within 2 meters proximity. First of all, the average received signal strength corresponding to the 2 meters distance mark is chosen as a threshold. When received signal strength is greater than the threshold, two radios are recognized as neighbors. Figure 7 (b) shows the probability that two radios are detected as neighbors at different distances. For nodes that are more than 2 meters apart, there is still a small $20 \%$ probability that they will be incorrectly detected as neighbor nodes. When two radios are less than 2 meters, there will be $71.6 \%$ probability that they are correctly detected as neighbors. 


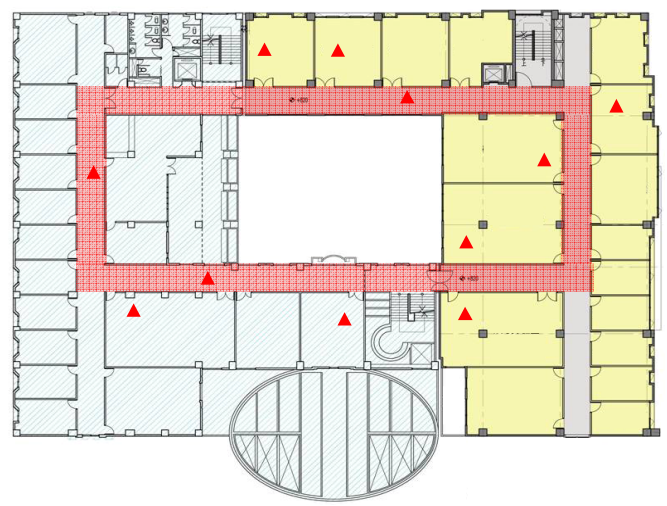

Fig. 6. Floor layout for the experiments. The red triangles are locations of IEEE 802.11 AP and the rectangular area is the corridors of the floor.

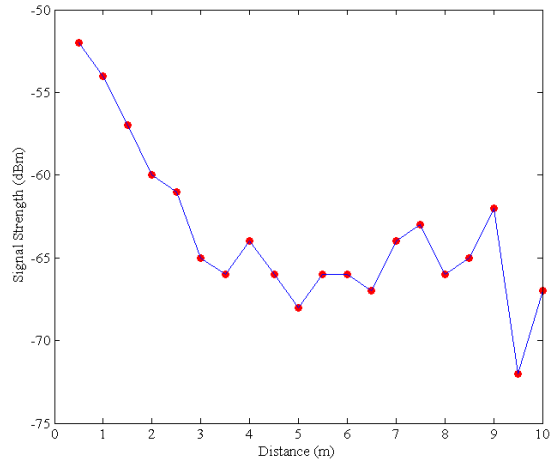

(a)

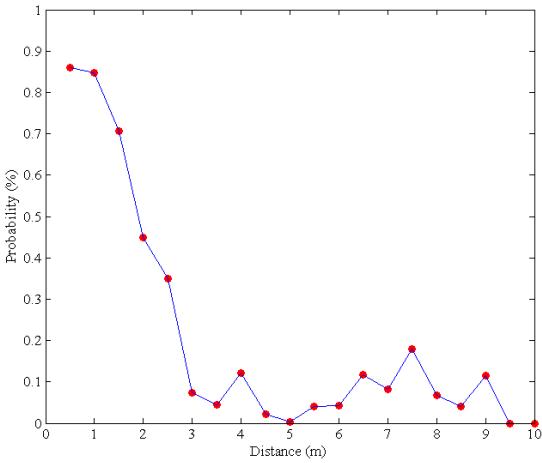

(b)

Fig. 7. Distance and the error model: (a) The relationship between distance of two ZigBee radios and the received signal strength, and (b) the relationship between distance of two ZigBee radios and the probability that they will be detected as neighbors.

We have found several sources of errors in the ZigBee-based neighborhood detection. The first source of error occurs when two nearby persons standing back-to-back, their neighboring links will not be detected, because ZigBee radio signal does not penetrate human bodies well. The second source of error occurs when a third person comes between two persons standing face-to-face, again their neighboring links will also be broken due to human body interference from the third person.

\subsection{Performance Evaluation}

To evaluate performance of our collaborative localization, we have designed two scenarios for experiments. The first scenario consists of stationary people forming stationary clusters. Locations of stationary clusters are pre-arranged shown in Figure 3 . We 
then observe how well our collaborative localization can improve positioning accuracy over a baseline $\mathrm{WiFi}$ positioning engine. The second scenario consists of mobile people forming mobile clusters. Movements of people are modeled after an ordinary group visit shown in Figures 12 and 15. In addition, two persons standing at two fixed locations on a corridor act as stationary passers. We then observe collaborative localization on how well stationary passers can help improving positioning accuracy of mobile clusters.

In the experiments, we simulate the error induced from ZigBee. The error is then appended to the ground truth to evaluate our collaborative location system. This is the preliminary study of deploying ZigBee as proximity sensor.

\subsection{Stationary Clusters (Scenario I)}

Scenario I consists of two cases of 3-person and 7-person stationary clusters with a cluster radius fixed to either 0.5 meter (called a dense cluster) or 1 meter (called a sparse cluster). Figure 8 plots cumulative distribution functions (CDF) of average positioning errors for 3-person dense and sparse clusters. Curves labeled "nocollaboration" show results when collaboration is not applied to location estimations, whereas curves labeled "collaboration" show results when collaboration is applied to location estimations. In addition, the curve labeled "3-person non-clustering" shows results when 3 stationary persons are standing apart without forming any cluster. This is used as a reference line for comparing with clustering cases. In the 3-person sparse cluster case, collaboration produces $37.2 \%$ accuracy improvement from 3.38 meters (no-collaboration) to 2.12 meters at $75 \%$ precision. Moreover, the average error is reduced by $34 \%$ from 2.41 meters to 1.59 meters. In the 3-person dense cluster case, collaboration produces $38 \%$ accuracy improvement from 5.38 meters (nocollaboration) to 3.34 meters at $75 \%$ precision. Moreover, the average error is reduced by $28.2 \%$ from 3.33 meters to 2.39 meters.

Figure 9 shows a positive relationship between the amount of accuracy improvement received by a target node, after applying collaboration, and $\triangle$ Confidence, which is the difference in confidence scores between a target node and its neighbor node, in a 3-person dense cluster case. The plot shows that when $\triangle$ Confidence is positive (i.e., a neighbor node has a higher confidence score than a target node), collaboration can help improving positioning accuracy of a target node. More importantly, a larger $\triangle$ Confidence results in a higher accuracy improvement, because a target node can benefit more from a neighbor node whose location estimation has a better accuracy than its location estimation. On the other hand, when $\triangle$ Confidence is negative (i.e., a neighbor node has a lower confidence score than a target node), collaboration is disabled because a neighbor node is likely to have worse positioning accuracy than a target node.

Figure 10 plots cumulative distribution functions (CDF) of average positioning errors for 7-person dense and sparse clusters. Results show that the amount of accuracy improvement in 7-person clusters is greater than that of 3-person clusters. In the 7-person sparse cluster, collaboration produces $54.7 \%$ accuracy improvement from 6.26 meters (no-collaboration) to 2.83 meters at $75 \%$ precision. Moreover, the average error is reduced by $49 \%$ from 4.20 meters to 2.14 meters. In the 7 -person 


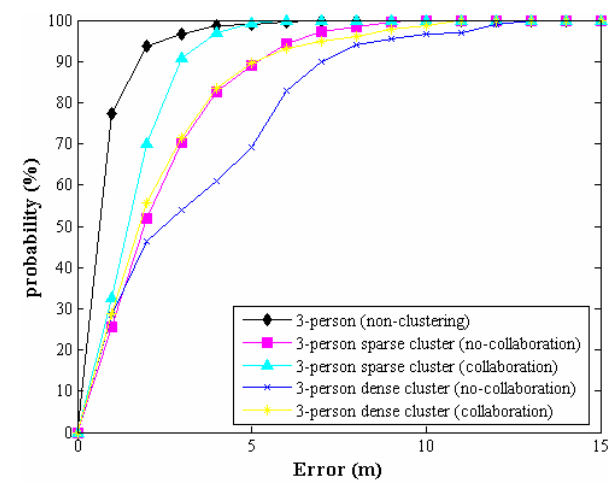

Fig. 8. CDF of average positioning errors in the 3 -person cluster scenario

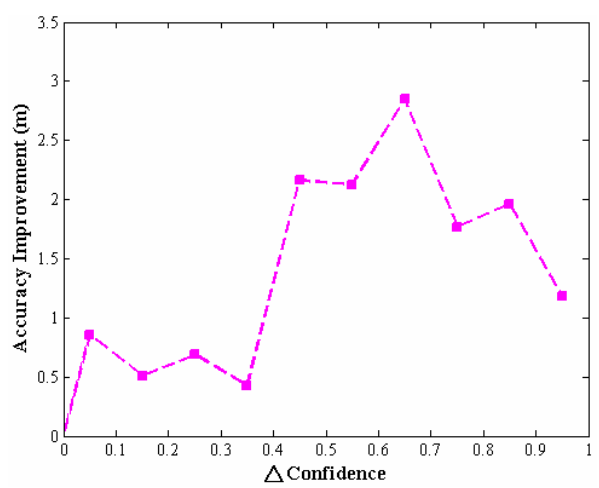

Fig. 9. The amount of accuracy improvement versus the neighborhood confidence difference in the 3-person dense cluster

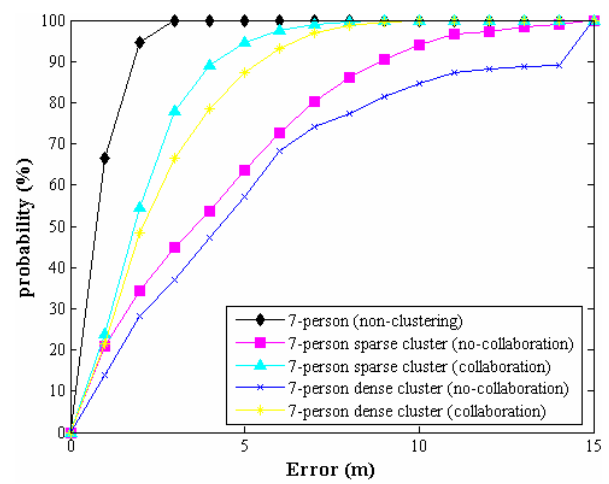

Fig. 10. CDF of average positioning errors in the 7-person cluster scenario

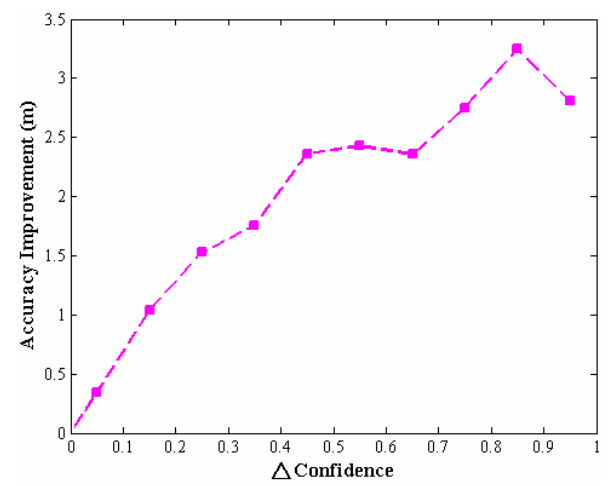

Fig. 11. The amount of accuracy improvement versus the neighborhood confidence difference in the 7-person dense cluster

dense cluster, collaboration produces $49.2 \%$ accuracy improvement from 7.25 meters (no-reduced by $56.3 \%$ from 5.95 meters to 2.60 meters. Similar to the 3 -person case, Figure 11 shows a positive relationship between the amount of accuracy improvement and the confidence difference with a neighbor node.

\subsection{Mobile Clusters (Scenario II)}

Scenario II consists of two cases of 4-person and 5-person mobile clusters with a cluster radius fixed to 0.5 meter. In addition, one more person in a 4-person case and two more persons in a 5-person case stand at fixed locations on a corridor as stationary passers. These settings are shown in Figure 12 and Figure 15. The total distance of the corridor is about 30 meters. Figure 13 plots cumulative distribution functions (CDF) of positioning errors for the stationary passer and average errors of the 


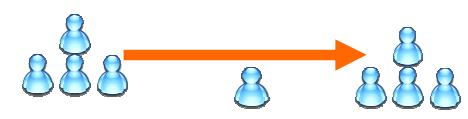

Fig. 12. 4 clustered persons walking by a stationary passer

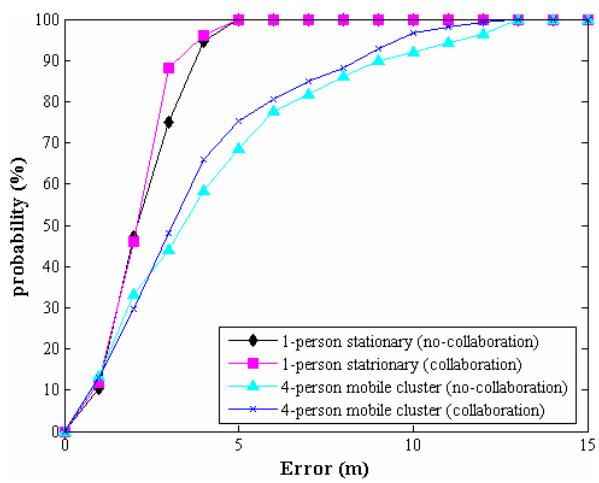

Fig. 13. CDF of average positioning errors in the 4-person mobile cluster case

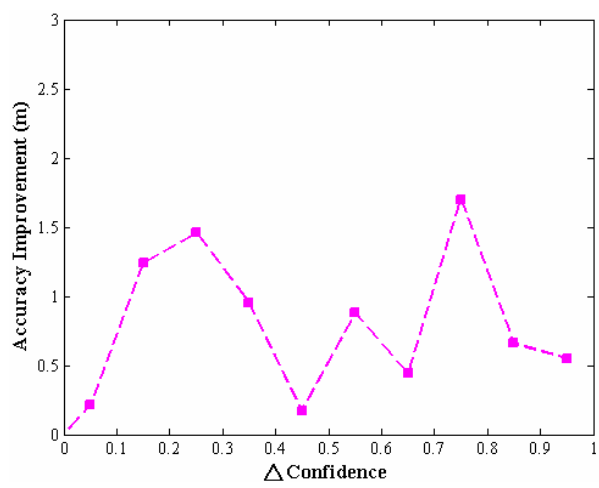

Fig. 14. Average of accuracy improvement in the 4-person mobile cluster case

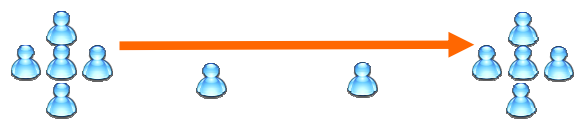

Fig. 15. 5 clustered persons walking by 2 stationary passers

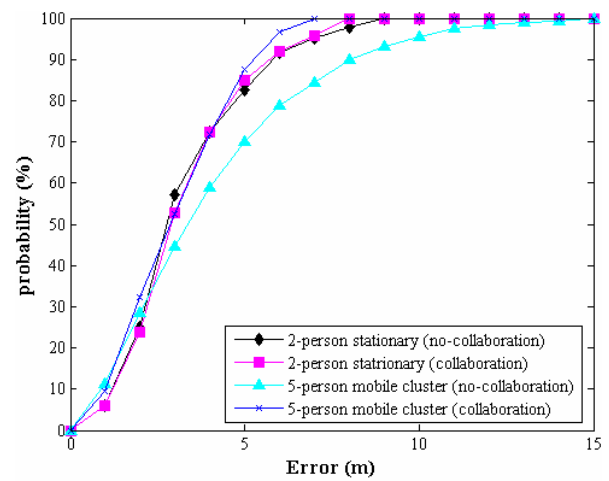

Fig. 16. CDF of average positioning errors in the 5-person mobile cluster case

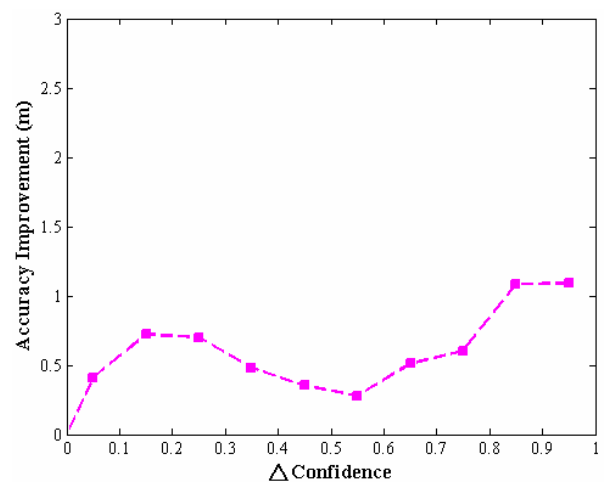

Fig. 17. Average of accuracy improvement in the 5-person mobile cluster case

4-person mobile cluster. The curve labeled "1-person stationary" shows positioning error of the stationary passer, and the curve labeled "4-person mobile cluster" shows average positioning error of a 4-person mobile cluster. In addition, curves labeled " no-collaboration" show results when collaboration is not applied to location estimations, whereas the curves labeled "collaboration" show results when collaboration is applied to location estimations. For the 4-person mobile cluster, the collaborative localization produces $16 \%$ accuracy improvement from 5.67 meters (nocollaboration) to 4.76 meters at $75 \%$ precision. Moreover, the average error is reduced by $17.5 \%$ from 4.57 meters to 3.77 meters. Figure 14 shows the relationship between 
the amount of improvement in positioning accuracy received by a target node and $\triangle$ Confidence. Results show a target node can benefit when collaborating with a higher confident neighbor node. However, we have found that accuracy improvement in mobile clusters is smaller than accuracy improvement in stationary clusters. The reason is that mobile nodes in general receive less accurate location estimations from a localization engine than stationary nodes; therefore, a node in a mobile cluster has a smaller chance of finding a high confidence neighbor node for collaboration than a node in a stationary cluster.

Figure 16 plots cumulative distribution functions (CDF) of positioning errors for two stationary passers and average errors of a 5-person mobile cluster. Results show that the amount of improvement in a 5-person mobile cluster is greater than that of a 4-person mobile cluster. For the 5-person mobile cluster, collaboration produces 26.2\% accuracy improvement from 5.58 meters (no-collaboration) to 4.12 meters at $75 \%$ precision. Moreover, the average error is reduced by $33.5 \%$ from 4.03 meters to 2.68 meters. Figure 17 shows the amount of improvement in positioning accuracy received by a target node and $\triangle$ Confidence. Results also show that a target node can benefit when collaborating with a higher confident neighbor node.

\subsection{Evaluation of Confidence Estimator}

We compare the accuracy improvement between our confidence estimation method, calculated from Equation (1), and two alterative estimation methods called uniform and random. Results, shown in Figure 18, plot cumulative distribution functions (CDF) of average positioning errors for a 7-person dense stationary cluster over three confidence estimation methods. Curves labeled "no-collaboration" shows a reference line when collaboration is not applied to location estimations. In the uniform method, every node receives equal confidence scores regardless of estimation errors from the underlying location engine. In the random method, a random number between 0 and 1 is assigned to each node as its confidence score, again regardless of estimation errors from the underlying location engine. In a 7-person stationary dense cluster, our method outperforms both uniform and random methods. Note that both random and

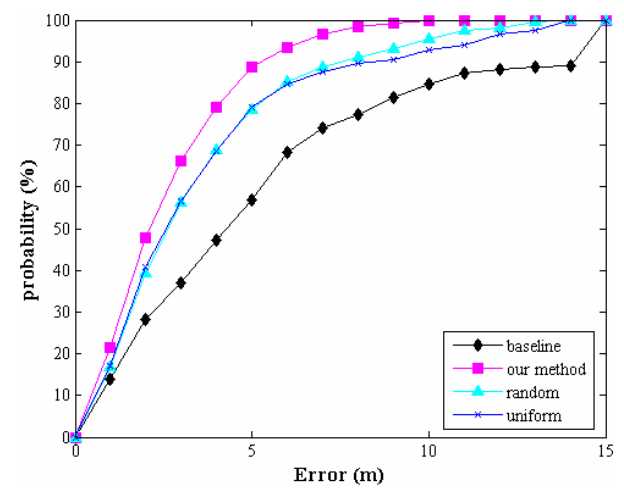

Fig. 18. Comparison of three confidence estimation methods (our current method, uniform, and random) in a 7-person, stationary, dense cluster 
uniform methods can still benefit from collaboration. The reason is that positioning errors from the underlying Ekahau location engine in general follow a symmetric distribution. Symmetric distribution means that while some location estimations of nodes are off in one direction, some location estimations of nodes in the same cluster are off in an opposite direction. Therefore, applying collaboration has a beneficial effect of error cancellation within a cluster of nodes.

\section{Related Work}

There has been extensive research on indoor localization. WiFi-based location systems can be either deterministic [5-8] or probabilistic [1-4] in matching RSSI frommobile devices to a radio map. The best systems claim $90 \%$ accuracy with an error of less than 1 2 meters. Some of these systems achieve better accuracy by combining different localization methods. That is, a hybrid system can benefit under situations where one method works poorly while another still works well. For example, Graumann et al. [9] aim at designing a universal location framework by using GPS in an outdoor environment, WiFi for an indoor environment, and Motes for proximity detection. Gwon et al. [10] proposed algorithms combining WiFi and Bluetooth sensors as information sources and selectively weighting them such that error contribution from each sensor can be minimized to improve the positioning accuracy. However, these systems do not address the human clustering problem.

Dynamic environmental factors can incur location estimation errors in traditional Wi-Fi location systems. Some proposed methods have attempted to address this issue. The temporal prediction approach in [11] can observe and learn how a radio map changes over time by employing emitters and sniffers to observe Wi-Fi RSSI variations. By applying regression analysis, the temporal prediction approach can learn the temporal predictive relationship between RSSI values received by sniffers and those received by target mobile devices. However, the temporal prediction approach assumes that changes in the environmental factors follow some predictable temporal patterns. However, in the people clustering case, such assumption does not hold given that people clustering is highly dynamic with individual node experiencing large variations of degradation. Reference points and regression model would not be able to capture such dynamic variations. Chen et al. [12] also addressed the challenge of providing stable position estimation under different environmental dynamics, including change of floor layout, change in relative humidity, and moving people. Since major floor layout changes occur less frequently, they can be managed by rebuilding a selective part of a radio map. To adapt to different relative humidity levels, a separate radio map can also be constructed for each humidity level. However, coping with moving people is more challenging, because it is infeasible to model and enumerate all possible cases of human clustering formations; furthermore, to construct different radio maps.

The idea of utilizing neighbor information to help localization is also used in sensor network localization and network coordination. DOLPHIN [13] deployed fixed nodes with ultrasonic and RF sensors in an environment. Nodes with known location coordinates are called master nodes. Non-master nodes can compute their relative locations to multiple master nodes by exchanging ultrasonic and RF signals. After 
performing iterative triangulation, nodes can get their absolute coordinates and become master nodes. He et al. [17] proposed a cost-effective, range-based localization approach called APIT for large scale sensor networks. Like the DOLPHIN system, the sensor network contains anchor devices that can obtain their locations through GPS receivers. Anchor nodes first broadcast their locations to non-anchor nodes. A non-anchor node then iteratively chooses different combination of 3 received anchor nodes and performs a Point-In-Triangulation (PIT) Test, which is used to determine whether a non-anchor node is inside a triangular region formed by 3 anchor nodes. If a non-anchor node resides in that triangular region, that region is marked as a possible location of the non-anchor node. After all combinations are exhausted, the center of intersections from all possible regions is calculated to estimate a non-anchor node's location. AFL [18] is a fully decentralized, anchor-free approach, utilizing the idea of fold-freedom to build a topology of a sensor network through local node interactions. In AFL, nodes start from a random initial coordinate assignment. By applying massspring optimization repeatedly, nodes' location estimations can converge to be near their true coordinates. Our work differs from these systems in that they assume nodes with known locations are stationary, whereas our work assumes that nodes are mobile people. In addition, these sensor network location systems assume that nodes with a cluster will not interfere with each other's positioning accuracy. However, in our system, people clustering results in blocked signals and degradation in positioning accuracy.

$\mathrm{Hu}$ et al. [14] proposed a method for sensor network localization that allows some node mobility. Seeds are nodes with known locations. Non-seed nodes apply a motion model to predict their locations by sensing whether they are entering proximity of or departing from a seed node. Seeds and non-seed nodes can be either mobile or stationary. However, their work does not consider errors in seed nodes' locations. This differs from our work in which node clustering leads to localization errors.

Neighbor information has also been used in network coordinate system at the Internet scale. GNP [15] places nodes with known locations in a network as landmark nodes. Other nodes can compute their approximate coordinates based on network round trip time (RTT) measurements to these landmark nodes. Vivaldi [16] proposed an alternative method without using landmarks. Each node in the network measures RTTs, translated into relative distances, to its neighbors and gradually converges to a virtual coordinate. However, these systems differ from our work in that they are not concerned with high location accuracy.

\section{Conclusion and Future Work}

This paper targets the problem of human clusters in WiFi-based localization. We have conducted tests to show that a human cluster can block WiFi signals, resulting in degradation of location accuracy. To address this issue, we have designed and implemented collaborative localization, which a node with a higher confidence of accuracy can help fine-tune location estimations of neighbor nodes that have a lower confidence. The proposed method consists of the three modules: (1) Neighborhood Detection finds nearby neighbors, (2) Confidence Estimation measures the accuracy of the location estimation, and (3) Collaborative Error Correction uses neighbor nodes' information to reduce the target node's positioning error. Our experimental results 
have shown that collaborative localization produces $28.2 \sim 56 \%$ accuracy improvement in stationary human clusters and 16 33.5\% accuracy improvement in mobile human clusters.

For future work, we would like to improve the accuracy of our neighborhood detection by using existing Wi-Fi adaptors or exploring alternative proximity sensors. One possible approach is to set WiFi adaptors in an ad-hoc mode for detecting signals strength from neighbor nodes. The advantage of this approach is that it requires no additional hardware. However, the challenge is in accuracy, considering that human body can cause interference to WiFi signals. To address this issue, we are looking for neighborhood detection methods that are not only based on using a single signal strength threshold.

Another future work is to place fixed anchor nodes in the environment where human clusters are likely to occur, e.g., in front of popular exhibits. These anchor nodes, with maximum confidence scores, can help fine-tune any nearby human clusters that border them.

\section{Acknowledgments}

This work was partially supported by grants from Taiwan NSC, Quanta Computer, and Intel (\#94-2218-E-002-055, \#94-2622-E-002-024, \#94-2218-E-002-075, and \#942218-E-002-057).

\section{References}

1. Paramvir Bahl and Venkata N. Padmanabhan: RADAR: An in-building RF-based user location and tracking system. IEEE INFOCOM (Mar. 2000) 775-784

2. ITRI, http://www.itri.org.tw

3. Paramvir Bahl, A. Balachandran, and V. N. Padmanabhan: Enhancements to the RADAR user location and tracking system. Technical report of Microsoft Research (Feb. 2000)

4. Ekahau. http://www.ekahau.com

5. V. Seshadri, G. V. Zaruba, and M. Huber: A Bayesian Sampling Approach to In-door Local-ization of Wireless Devices Using Received Signal Strength Indication. IEEE Conference on Pervasive Computing and Communications (PerCom) (Mar. 2005)

6. J. Hightower, and G. Borriello: Particle Filters for Location Estimation in Ubiquitous Computing: A Case Study. International Conference on Ubiquitous Computing (Sept. 2005)

7. D. Schulz, D. Fox, and J. Hightower: People Tracking with Anonymous and ID-Sensors using Rao-Blackwellised Particle Filters. International Joint Conference on Artificial Intelligence (Aug. 2003) 921-926

8. D. Fox, J. Hightower, L. Liao, D. Schulz, and G. Borriello: Bayesian Filtering for Location Estimation. IEEE Pervasive Computing, vol. 2, no. 3 (July-Sept. 2003) 24-33

9. David Graumann, Jeffrey Hightower, Walter Lara, and GaeTano Borriello: Real-world Implementation of the Location Stack: The Universal Location Framework. IEEE Workshop on Mobile Computing Systems \& Applications (WMCSA) (Oct. 2003)

10. Youngjune Gwon, Ravi Jain, and Toshiro Kawahara: Robust Indoor Location Estimation of Stationary and Mobile Users. IEEE INFOCOM (Mar. 2004)

11. J. Yie, Q. Yang, L. Ni: Adaptive Temporal Radio Maps for Indoor Location Estimation. International Conference on Pervasive Computing (May 2005) 
12. Y.C. Chen, J.R. Chiang, H.H. Chu, Polly Huang, and A. W. Tsui: Sensor-Assisted Wi-Fi Indoor Location System for Adapting to Environmental Dynamics. International Symposium on Modeling, Analysis and Simulation of Wireless and Mobile Systems (Oct. 2005)

13. Masateru Minami, Yasuhiro Fukuju, Kazuki Hirasawa, Shigeaki Yokoyama, Moriyuki Mizumachi, Hiroyuki Morikawa, and Tomonori Aoyama: DOLPHIN: A Practical Approach for Implementing a Fully Distributed Indoor Ultrasonic Positioning System. International Conference on Ubiquitous Computing (UbiCom) (Sep. 2004) 347-365

14. Lingxuan $\mathrm{Hu}$ and David Evans: Localization for Mobile Sensor Networks. International Conference on Mobile Computing and Networking (MobiCom) (2004)

15. T. S. Eugene Ng and Hui Zhang: Predicting Internet Network Distance with CoordinatesBased Approaches. IEEE INFOCOM (2002) 170-179

16. Frank Dabek, Russ Cox, Frans Kaashoek, and Robert Morris: Vivaldi: A Decentralized Network Coordinate System. ACM SIGCOMM (2004)

17. Tian He, Chengdu Huang, Brian M. Blum, John A. Stankovic and Tarek Abdelzaher: Range-Free Localization Schemes for Large Scale Sensor Networks. ACM International Conference on Mobile Computing and Networking (MOBICOM) (2003) 81-95

18. Nissanka B. Priyantha, Hari Balakrishnan, Erik Demaine, and Seth Teller: Anchor-Free Distributed Localization in Sensor Networks. ACM International Conference on Embedded Networked Sensor Systems (SenSys) (2003) 340-341 